\title{
Assessment of Handwriting Skill in South Indian Children with Developmental Coordination Disorder
}

\author{
Ganapathy Sankar $\mathbf{U}^{1}$ and Monisha $\mathbf{R}^{2 *}$ \\ ${ }^{1}$ Professor, SRM College of Occupational Therapy, India \\ ${ }^{2}$ Assistant Professor, SRM Institute of Science and Technology, India \\ *Corresponding author: Monisha R, Assistant Professor, SRM College of Physiotherapy, SRM Institute of Science and Technology, Chennai, India
} Submission: 眥August 13, 2018; Published: 阱 October 30, 2018

\begin{abstract}
Background: Children with developmental coordination dis $\urcorner$ order (DCD) experience difficulties produc-ing controlled and coordinated movement and this difficulty interferes with their activity of daily living and academic achievement.

Objective: The purpose of this study was to characterize handwriting deficits in children with developmental Coordination disorder (DCD).

Methods: 10 boys with DCD with age ranges from 6 to 8 years with Kaufman Brief Intelligence Test (K-BIT) scoring above 80 were included in the study after having satisfied the Diagnostic and Statistical Manual of Mental Disorders for DCD. Children with pervasive developmental disorder were not included in this study.
\end{abstract}

Result: The attainment of handwriting skill by DASH test was markedly slower in children with DCD, who have difficulties in writing within an appropriate time frame and when writing complex character.

Conclusion: Children with DCD have difficulties performing the open-loop and closed-loop movements required for fluent handwriting.

\section{Background}

Children with developmental coordination disorder (DCD) have difficulties in coordinated activities [1]. The impaired motor coordination has significant implications in academic achievement and in activities of daily living. Several researchers have identified the etiology of DCD as a part of cerebral palsy but till date etiology of DCD is unknown. According to American Psychiatric Association, failure of acquisition of motor skills, which is not on the basis of impaired learning difficulty and lack of exposure to opportunity to gain motor skills as their peers and they should not have any other diagnosable neurological, psychiatric, or general medical condition for the global problems observed in motor performance [2]. The condition is more common in boys than girls and affects $5 \%$ to $6 \%$ of children and higher percentage of DCD is found among the children with the history of prenatal difficulties [3]. There has been much difficulty in predicting the percentage of prevalence rate in DCD as the population group is selected in different ways and the prevalence is directly related to the manner in which the assessment tool is employed and hence there is instability in identifying the prevalence. It is apparent that children will have difficulty in estimating body position and other proprioceptive skill, these problems will affect their ability to learn new task. Children's were less able to match eye movements to a moving target [4].
Exploration of range of perceptual deficit in task requiring motor control, learning and cognition. Multiple difficulties are associated with DCD, but each child experience distressing difficulty in a unique task [5]. Frequently children with DCD have reported difficulty to follow motor commands, inability to participate in physical activity with peer groups, difficulty in sequencing task and increase in time to complete a minor task and difficulty with handwriting and learning [6]. If proper identification of DCD is not undertaken, time and money is wasted for these group of children and they will be receiving inappropriate therapy and fail to achieve any positive result and all the children with coordination disorder will pass from one service to next to achieve good result in academics and in physical activity [7]. If this population is left untreated, adolescents with DCD have been shown to have psychiatric symptoms to social negativism [8].

Difficulties and clinical presentation are unique to each child with DCD. Evaluating the suitable intervention for these children is always a challenge to the health care practitioners. The cause of the condition is undetermined and various researchers have identified many subtypes in ICD, which is still under discussion. Many practitioners employ a wide variety of treatment approaches for these children. But none showed to be effective in correcting 
all their difficulties in motor performance. Even though bottom up approaches are frequently used in DCD children's, but there is still a lag in meeting the demands and difficulties met in children's with DCD. Functional movement pattern is believed to be emerged from complex interaction of multiple subsets. Contemporary theories have discussed regarding the emergence of functional movement pattern from interaction of multiple subsystems [7]. DCD children when attempted to perform motor task, they repeatedly perform the same task, regardless of any, improvements. They experience problem with planning a new motor task and integrating impulse to identify appropriate cognitive strategies to resolve problems faced with children of DCD. Thus, the problems with DCD children were difficulties in learning, sequencing events and in performance of motor skills, learning has been affected in these children is because of difficulties in planning intervention. It is estimated that school going children experience handwriting difficulties. handwriting is related to writing speed and pen, pencil holding style and handwriting instruction has been given to every school child is an important element in preventing handwriting difficulties. performance in the classroom and during test hour is the key factor in identifying children with handwriting problem and observing children struggling to complete the written test paper within the stipulated time period, handwriting problems are evident when the children's were assigned a task to be completed within the time frame, although some studies have used teachers identification in children facing handwriting difficulty in classroom, but the teachers perception is not reliable and it is biased as every teacher will have their own perception on the initial observation and the uniformity in perception is varied when multiple observations made by the same and with different teachers [9]. Hand writing performance was measured by finger-succession task. Here the fine motor movements have been employed to complete the test and this is the best indicator of handwriting difficulty. Hence the assessment of fine motor control in handwriting evaluation is needed.

By visual inspection it is impossible to rate the difficulties experienced in handwriting performance by children with DCD. Fine motor movements of the hand are crucial to perform handwriting task, because handwriting involves a complicated sequences of fine motor movements. Motor in coordination is related with problems in academic achievement in children with DCD. When evaluating the Academic performance in DCD most directly affected component is handwriting skill. As a result, poor handwriting has become one of the important diagnostic criteria for DCD.

\section{Method}

Convenient sampling was used to recruit 10 boys with DCD. The children aged ranged from 6 to 8 years and the boys were included in the study after having satisfied the Diagnostic and Statistical Manual of Mental Disorders criterion (American Psychiatric Association) for DCD. Markedly impaired motor coordination, evidenced by scoring below the $15^{\text {th }}$ percentile on the Movement Assessment Battery for Children (M-ABC) difficulties with functional activities of daily living and in academics, scoring above 80 on the Kaufman Brief Intelligence Test (K-BIT) children with pervasive developmental disorder were not included in this study

\section{Procedure}

For 6 weeks, each child handwriting skill and task assigned were videotaped and all the children has participated in 12-hour session, as he two sessions per week, received the individually tailored treatment protocol structured for each children with DCD. The children identified practicing handwriting as difficult task. Each child worked on his own 1 hour of handwriting practice session at every week. These sessions were conducted in the presence of the experimenter. Treatment session has been delivered after signing consent from the parent and practicing sessions occurred with one parent in presence, in a private therapy room or outdoors as dictated by the demands of the activity. Videotape data provides a permanent record of the child performance that can be reviewed in future to compare the improvised handwriting task and time taken to complete the task with documented data as videotaped by the experimenter. On comparing the field observation, videotape data provides reliable data resource. VMI score has been obtained in the baseline, various Studies done have indicated that VMI correlates with handwriting performance and it is useful evaluation tool in the screening of handwriting dysfunction. 5 boys had handwriting deficit without DCD as assessed in accordance with MABC and hence they were excluded out of the study. Handwriting assessment was done with an A4 blank paper was fixed on to the table for initiating the handwriting tasks, the child was seated comfortably with hips and knees flexed at 90 to 95 degrees and elbows were flexed according to the comfort of the child and with forearms resting comfortably on the table. The tasks were presented on paper near the table in 24-point Times new roman font type. Examiner gives instruction to child and they were asked to write according to their own speed and there is no time constrain. DASH was used to examine the speed in handwriting component and to examine the handwriting product, assessing speed of performance on a range of writing tasks. It also includes free-writing task and examine a range of different types of handwriting like copying. The total number of words completed by a child per minute was used to analyze the relationship between visual perception and handwriting speed. DASH task has been administered to all the children and four main components has been tested, in copy best task, all the children were asked to copy down the sentence in their own handwriting and the same sentence has to be repeated by the child for two minutes. Total number of words completed by a child per minute was recorded, before scoring incomplete words, punctuation marks and the words which were written illegible were excluded out. Second task assigned to the child was Copy Fast, here the child has been asked to write alphabets or numbers from memory for one minute, at the end of the test, every word should be readable. The number of correctly sequenced letters per minute was recorded. Third task was on writing alphabets, forth task assigned to the child was Freewriting, here the child has been asked to write his topic of interest and they were given with a topic if they find difficulty in finding what to write about? Here in this test, content of the free writing is not assessed, but the continuity in writing for 10-min without any difficult was documented. Total number of words he has completed at the end of 10 minutes was evaluated. Handwriting legibility has been assessed using HLS. 


\section{Data Analysis}

Figure 1-3 and Table $1 \& 2$

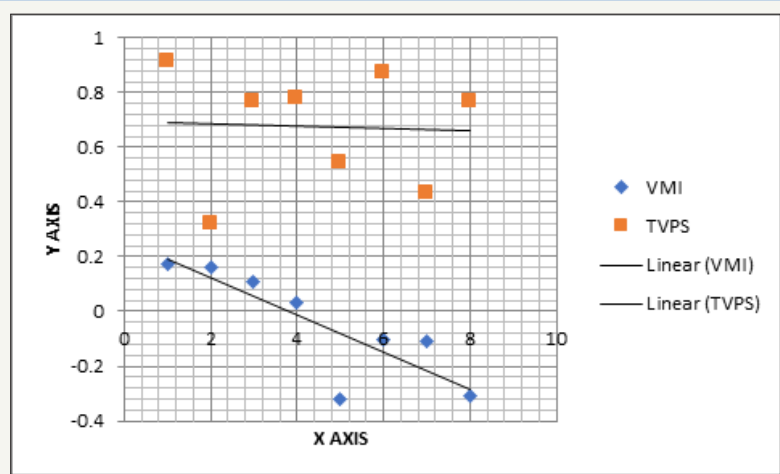

\section{Figure 1}

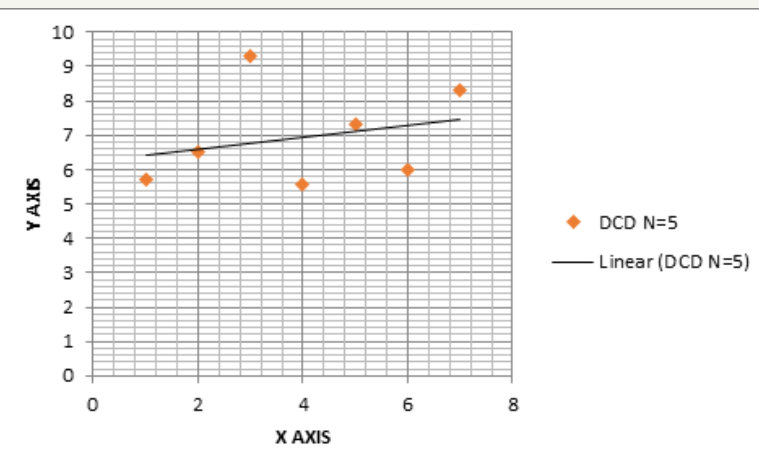

Figure 2

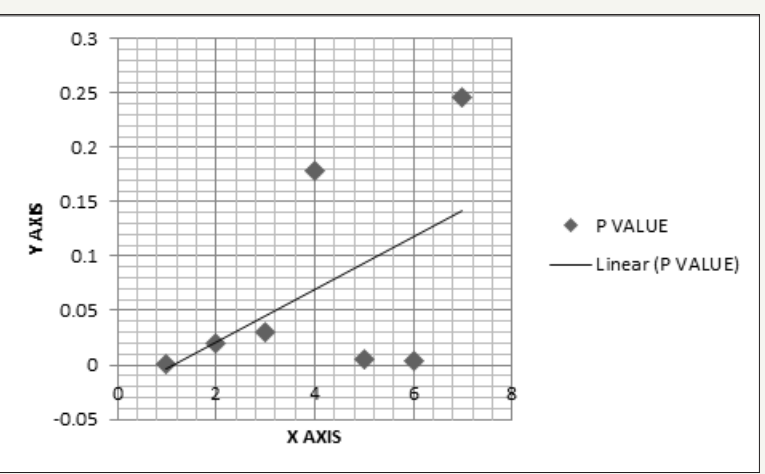

Figure 3

Table 1: Test for visual perception skill.

\begin{tabular}{|c|c|c|c|}
\hline S. No & TVPS Sub Test & DCD N=5 & P Value \\
\hline 1. & Visual Dicrimination & 5.70 & 0.001 \\
\hline 2. & Visual Memory & 6.50 & 0.020 \\
\hline 3. & Spatial Relationship & 9.30 & 0.030 \\
\hline 4. & Form Constancy & 5.55 & 0.178 \\
\hline 5. & Sequencial Memory & 7.30 & 0.005 \\
\hline 6. & Visual Figure-Ground & 6.00 & 0.004 \\
\hline 7. & Visual Closure & 8.32 & 0.246 \\
\hline
\end{tabular}

Table 2: Correlation between VMI and TVPS on DCD children.

\begin{tabular}{|l|l|l|l|}
\hline S. No & Handwriting Speed & VMI & TVPS \\
\hline 1. & Copy Best & 0.174 & 0.911 \\
\hline 2. & Copy Fast & 0.160 & 0.321 \\
\hline 3. & Alphabets & 0.111 & 0.765 \\
\hline 4. & Free Writing & 0.033 & 0.777 \\
\hline 5. & Copy Best $\%$ & -0.322 & 0.543 \\
\hline 6. & Copy Fast $\%$ & -0.100 & 0.875 \\
\hline 7. & Free Writing & -0.111 & 0.433 \\
\hline 8. & HLS & -0.311 & 0.766 \\
\hline
\end{tabular}




\section{Discussion}

Little research has been done in handwriting difficulties faced by children as much focus was given only for motor component, this led to lag in research in this patient population. Much debate has been on research in DCD children, shows that handwriting process and progress will be affected in all these children of same age group. Aim of this current study was on evaluation of visual perception and visual motor integration. In this study it was proved that DCD children performed significantly more poorly on the VMI test and TVPS. We also examined group differences in the subtests of the TVPS and found that the DCD group was poorer on all subtests with the exception of spatial relationships and form constancy. However, in the current study we examine the extent to which these measures were related to different aspects of handwriting performance. No significant correlations were found between the visual perceptual measures and the handwriting measures within the individual child received the intervention. Previous research showed a correlation between visual motor integration and handwriting legibility in their sample of children with handwriting difficulties. In our own sample we controlled for co-occurring deficits both through strict inclusion criteria and also by running additional analyses to ensure that cooccurring difficulties such as attention deficits did not impact on handwriting performance. In addition, this study considered a range of writing tasks and found no relationship between the visual perceptual measures and any of the handwriting measures.

Indeed, the International Guidelines for DCD provide a list of useful tools for assessing handwriting difficulties in children with DCD, all of which require the completion of a handwriting task rather than visual perceptual measures (Blank et al., 2012). The research in this study indicate that tests of visual motor integration and visual perception do not appear to be related to poor handwriting performance in children with DCD.

\section{Conclusion}

The Study concluded that children with DCD can learn simple sequential movements, but it would be interesting to examine form consistency and accuracy to ascertain whether more complex letter forms are more problematic than others. In relation to handwriting fluency, correct directional movements would be an important factor in increasing automaticity in handwriting. The findings of this study suggest that there are other factors which may explain handwriting difficulties in children with DCD and further investigation is needed.

\section{References}

1. Karlsdottir R, Stefansson T (2002) Problems in developing functional handwriting. Percept Mot Skills 94(2): 623-662.

2. Graham S, Harris KR, Fink B (2000) Is handwriting causally related to learning to write? Treatment of handwriting problems in beginning writers. J Educ Psychol 92(4): 620-633.

3. Graham S (1998) A review of handwriting scales and factors that ontribute to variability in handwriting scores. J Sch Psychol 24(1): 6371.

4. Chang SH, Yu NY (2005) Evaluation and classification of types of Chinese handwriting deficits in elementary school children. Percept Mot Skills 101(2): 631-647.

5. Sudsawad P, Trombly CA, Henderson A, Tickle-Degnen L (2001) The relationship between the evaluation tool of children's handwriting and teachers' perceptions of handwriting legibility. Am J Occup Ther 55(5): 518-523.

6. Berninger VW, Rutberg J (1992) Relationship of finger function to beginning writing: application to diagnosis of writing disabilities. Dev Med Child Neurol 34(3): 198-215.

7. Marquardt C, Mai N (1994) A computational procedure for movement analysis in handwriting. J Neurosci Methods 52(1): 39-45.

8. Ganapathy Sankar U, Saritha S (2011) A study of prevalence of developmental coordination disorder (DCD) at Kattankulathur, Chennai. Indian Journal of Physiotherapy and occupational therapy 5(1): 49-52.

9. Ganapathy Sankar U (2018) The prevalence of developmental coordination disorder at Kattupakkam, Tamilnadu. IOSR Journal of Pharmacy 8(2): 49-52.

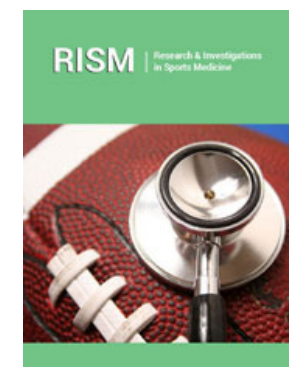

Research \& Investigations in Sports Medicine

\section{Benefits of Publishing with us}

- High-level peer review and editorial services

- Freely accessible online immediately upon publication

- Authors retain the copyright to their work

- Licensing it under a Creative Commons license

- Visibility through different online platforms 